\title{
Dark Matter and Gravitational Waves
}

\author{
Joseph J. Smulsky
}

Institute of Earth's Cryosphere, Tyum. SC of SB RAS, Federal Research Center, Tyumen, Russia

Correspondence to: Joseph J. Smulsky, jsmulsky@mail.ru

Keywords: Dark Matter, Gravitational Waves, Hypotheses, Interaction, Charged Particles, Newtonian Gravity

Received: February 18, $2021 \quad$ Accepted: March 19, $2021 \quad$ Published: March 22, 2021

Copyright $\odot 2021$ by author(s) and Scientific Research Publishing Inc.

This work is licensed under the Creative Commons Attribution International License (CC BY 4.0).

http://creativecommons.org/licenses/by/4.0/

\section{(c) (i) Open Access}

\section{ABSTRACT}

Two reviews of papers are considered. The first paper for a galaxy model uses matter consisting of neutrinos, bosons and other similar particles. It is shown that these particles were introduced as a result of an incorrect description of interactions in the Theory of Relativity. In reality, with the relative motion of interacting particles, their interactions force changes, and not their mass. It is shown that models of such stellar associations as globular clusters and galaxies should be created on the basis of the substance that exists on Earth. The second peer-reviewed paper proposes to create LIGO on the Moon. It is shown that gravitational waves do not exist. They were introduced to explain the excessive rotation of the Mercury's perihelion. However, the excessive rotation of the Mercury's perihelion is due to the Sun oblateness. The paper shows that gravitational waves, the Big Bang, the expanding Universe, dark matter, dark energy, etc. appeared on the basis of unfounded hypotheses. The urgent task is to eliminate them from science.

\section{INTRODUCTION}

The method of cognition of the surrounding world with the help of hypotheses has taken root in modern science. To explain any phenomenon, a hypothesis about its mechanism is put forward. On its basis, a ramified explanation of this phenomenon is built, i.e. its theory is developed. With the appearance of new data, this explanation contradicts them. To get rid of the contradiction, an additional hypothesis is introduced. In the future, new contradictions appear which are then eliminated by new hypotheses. And so the process has been going on for 100 years.

Let us give an example. When studying the light of distant astronomical objects, it was found that with an increase in the distance to them, its frequency decreases, i.e. the light turns more red. Such reddening of light, as well as a decrease in the frequency of sound, occurs with the distance of their sources from the observer. This phenomenon is called the Doppler Effect.

It was hypothesized that the reddening of the light of distant stars and other objects is due to the Doppler Effect. Therefore, these objects are moving away from us.

However, it turned out that this reddening of light occurs in all directions relative to the Earth. The 
second hypothesis was accepted that all stars are moving away from us, i.e. the whole Universe is expanding.

The expansion takes place in the future, and in the past, all objects must come closer, and the Universe will turn into a point. Therefore it is accepted the third hypothesis about the Big Bang, which resulted in the birth of the Universe.

However, it turned out that the further away an astronomical object is, the more reddening of its light, and, consequently, the greater the removal velocity. For example, for the quasar OH471, the relative shift of the wavelength $\lambda$ towards the red side is $\mathrm{z}=3.4$, and for the quasar GB1508 $+5714 \mathrm{z}=4.3$, where $z=\Delta \lambda / \lambda$. According to the classical Doppler Effect, in the first case, the quasar moves away with a velocity equal to 3.4 the speed of light, and in the second-4.3. For the Doppler Effect in the Theory of Relativity, different authors give different formulas. For quasar GB1508 +5714 , according to some of them, the removal velocity is 0.93 times the speed of light, and according to others, this quasar moves 1.13 times faster than light.

However, an increase in velocity is not possible. Since, in accordance with Newton's law of universal gravitation, bodies are attracted to each other; then after the explosion, the velocity of their removal will decrease, and not increase. Here, it would seem, it is necessary to abandon all these hypotheses. However, a fourth hypothesis about dark energy is accepted. Thanks to dark energy, bodies after the Big Bang accelerate, but not slow down. For example, a well-known expert on models of the early Universe offers the following explanation in his popular lectures. Nobody knows yet what dark energy is, what its properties are. But it certainly exists. Indeed, without it, there would be no expanding Universe and there would be no Big Bang.

This example shows what kind of understanding of the surrounding world appears as a result of the method of cognition using hypotheses. This method gives us an imaginary world, not a real one.

The followers and participants of creating such an imaginary world themselves, over time, begin to doubt its truth. Towards the end of their lives, they come to complete frustration. But new generations continue this process of world creation with enthusiasm. It may take years or decades to thoroughly study all the features of the phenomenon, their connections with other phenomena, to set up experiments and observations and perform cumbersome calculations; even a lifetime may not be enough. And a hypothesis can be put forward immediately and thus win worldwide recognition. Therefore, the creation of an imaginary world continues.

Within a few weeks of each other, two journals in the fields of astronomy and physics sent me papers by such participants in the creation of this world for review. Some extended reviews of these papers are offered to the reader's attention.

\section{MODELS OF GALAXIES}

The paper "Adding Dark Matter to the Standard Model" examines mathematical models of galaxies using various imaginary particles such as bosons, neutrinos of various types, etc. To explain the rotational velocity profile of galaxies, dark matter is used, consisting of these particles.

These particles are proposed by theoretical physicists when considering various models of the micro-world in the framework of the Theory of Relativity (TR). The hypothesis about the change in the mass of two relatively moving charged particles was introduced in the Theory of Relativity to explain their interaction.

However, the Theory of Relativity is erroneous $[1,2]$. The interaction of two such particles is determined by the force (1), which depends on the distance and velocity between them $[1,3-5]$. Therefore, the change in particle mass accepted in TR does not exist. All imaginary particles also do not exist [2]. Therefore, there is no dark matter, consisting of bosons, neutrinos and other such particles.

$$
\boldsymbol{F}_{i k}=\frac{q_{i} q_{k}\left(1-\beta_{i k}^{2}\right) \boldsymbol{r}_{i k}}{\varepsilon\left\{r_{i k}^{2}-\left[\boldsymbol{\beta}_{i k} \times \boldsymbol{r}_{i k}\right]^{2}\right\}^{3 / 2}},
$$


where $r_{i k}$ is the radius-vector from a particle with a charge $q_{k}$ to a particle with a charge $q_{\dot{p}}$

$\boldsymbol{v}_{i k}$ is the velocity of a particle with a charge $q_{k}$ in relation to a particle with a charge $q_{\dot{p}}$

$\boldsymbol{\beta}_{i k}=\boldsymbol{v}_{i k} / c_{1}$ is dimensionless velocity;

$c_{1}=c / \sqrt[i]{\mu \varepsilon_{d}}$ is the speed of light in the medium between particles;

$\varepsilon$ and $\mu$ are dielectric constant of the medium and its magnetic permeability, respectively;

$\left[\boldsymbol{\beta}_{i k} \times \boldsymbol{r}_{i k}\right]$ is the vector product $\boldsymbol{\beta}_{i k}$ and $\boldsymbol{r}_{i k}$.

The Theory of Relativity always considers the interaction of two particles. In reality, not two particles always interact, but several. Formula (1) expresses the force of action of particle $k$ on particle $i$. Moreover, each of them is influenced by the rest particles. Therefore, to describe the real interaction by the method of the Theory of Relativity, it is necessary to introduce as many changes in mass, time and distance associated with particle $i$ as there are particles $k$. These changes with particle $i$ must occur simultaneously. This shows how absurd of the Theory of Relativity is. This is first. And secondly, this also shows that the calculations of the interactions of charged particles in the Theory of Relativity are not correct.

In the paper "Adding Dark Matter to the Standard Model", a new type of substance is introduced. Meteorites coming to Earth and all space missions convince us that there are only bodies in space that consist of the same substance that exists on Earth. Stars, constellations and galaxies are built from this substance. It is necessary to consider models of galaxies based on such matter. A number of complex mathematical problems have been solved [6-9]. Methods for solving problems of interaction of many bodies have been created $[10,11]$. They allow creating deterministic models of globular star clusters (Figure 1) and galaxies [12]. These models will reveal the mechanism of interactions and movements of bodies in these objects.

The Theory of Relativity should be thrown away and forgotten $[1,2]$. It is also necessary to throw

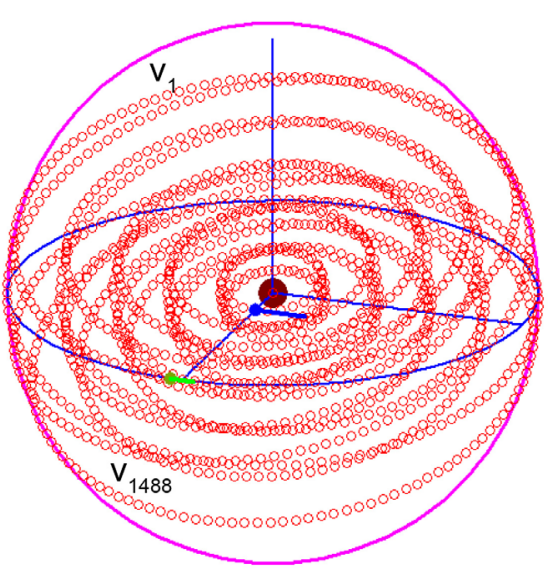

(a)

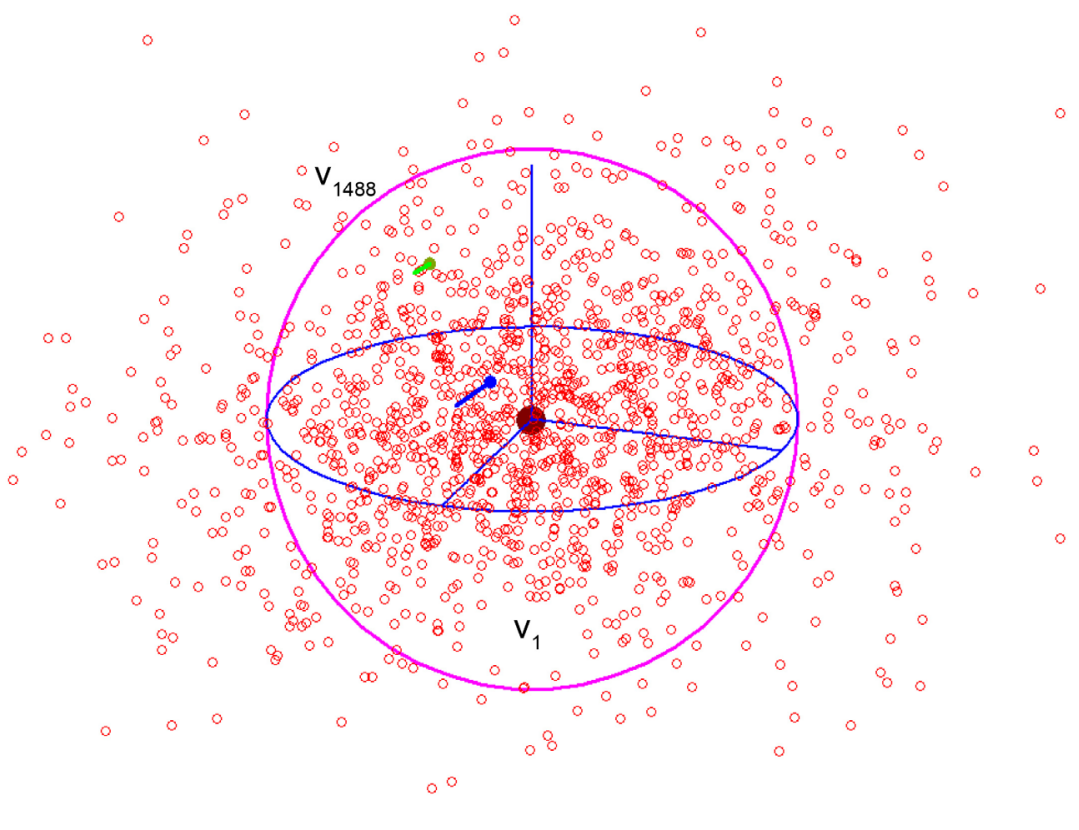

(b)

Figure 1. Multi-layer spherical model of a globular star cluster: shown are five layers involving 1488 peripheral bodies; (a) at the time the structure was formed; (b) after the 196-th revolution of the first-layer body; the line bars at bodies 1 and 1488 indicate their velocity vectors, $v_{1}$ and $v_{1488}$ [12]. 
away and forget all invented particles, as well as imaginary objects and phenomena, such as the Big Bang, the expanding universe, black holes, etc. $[2,13]$.

The paper "Adding Dark Matter to the Standard Model" presents a perverse understanding of the world around us. Therefore, it should not be published.

\section{GRAVITATIONAL WAVES}

The paper "A Lunar LIGO For NASA's Return To The Moon” proposes a Laser Interferometer Gravitational Wave Observatory (LIGO) on the Moon. It is designed to discover gravitational waves.

Such an observatory on the Moon has several advantages over an observatory on Earth. There is no atmosphere on the Moon and the force of gravity much less. Therefore, there is no need to make evacuated canals for the light beam and there are lower requirements for the strength of the LIGO structure. This will lead to significant reductions in the cost of creating LIGO and its operation.

In addition, the accuracy of LIGO is increased due to the possible increase in the length of the beam path. And together with the terrestrial LIGO, the length of the base can be increased to the radius of the lunar orbit.

The paper notes that after decades of studying the elusive phenomena predicted by Einstein's general theory of relativity, gravitational waves has been discovered. Therefore, it is important to continue this research and create LIGO on the Moon.

In reality, no gravitational waves have been detected. By the centenary of General Relativity, a paper by B.P. Abbott and more than 1100 of his co-authors was published [14], which allegedly provided evidence of the detection of gravitational waves. Elementary analysis shows that this paper is a gross hoax [2, section 5], [15, section 4], [16]. There is no evidence in this paper for the existence of gravitational waves.

There are no gravitational waves and cannot be. In General Relativity, they were introduced on the basis of the hypothesis that gravity propagates at the speed of light. This hypothesis was adopted to explain the excess of rotation of the Mercury's perihelion, equal to 43 arc seconds per century (arcsec/century). A. Einstein borrowed the equations and their solution from the work of Paul Gerber [17], which showed that at the luminal velocity of gravitation, the rotation of the perihelion of Mercury occurs by 43 arc$\mathrm{sec} /$ century. However, Paul Gerber's solution is wrong. The luminal velocity of gravitation gives the rotation of the perihelion 0.23 arcsec/century [1], and not 43 arcsec/century, i.e. in 187 times less.

Therefore, the luminal velocity of the gravity propagation cannot explain the excess of rotation of the Mercury's perihelion!

A more accurate analysis of observations showed that relative to motionless space, the excess of rotation of the Mercury's perihelion is 53 arcsec/century, and not 43 arcsec/century [18]. As a result of the interaction of bodies in the Solar system according to Newton's law of gravity, the Mercury's perihelion rotates at a speed of 530 arcsec/century [19], while according to observations this speed is equal to 583 arc$\mathrm{sec} /$ century. When solving the problem of the interaction of bodies in the Solar system, bodies are considered as material points. The action of a strictly spherical body is equivalent to the action of a material point. But the Sun is not spherical, but flattened. Taking into account the Sun's shape gives the missing before observations 53 arcsec/century $[12,18,20]$. A more detailed Addition for Mercury's perihelion is presented below. So, the calculated motion of the Solar system bodies, using Newton's law of gravity, completely coincides with observations $[12,18,20]$. Therefore, there is no need to introduce the luminal velocity of gravity propagation. General relativity, including gravitational waves, must be thrown away and forgotten.

The paper “A Lunar LIGO For NASA's Return To The Moon”, offering meaningless work, should not be published.

For future flights to the Moon, necessary and useful research needs to be planned. It is necessary to study the geography and geology of the Moon. It is necessary to study the Earth, the Sun and outer space from the Moon. It is necessary to study the flow of solar substance on the Moon, as well as its content in the lunar surface. These results will open up a lot of new things in understanding not only outer space, but 
also in understanding many phenomena on Earth. Such results will be beneficial to humanity.

There is no need to waste energy and money on worthless tasks due to delusion. As an example of one such problem, one can cite the installation of corner reflectors on the Moon. The funds were spent, but there is no effect from them.

This also applies to the mentioned in the paper laser interferometer space antenna (LISA) of the European Space Agency (ESA), which is also designed to detect gravitational waves. This 400 million euros verification mission has been completed but will continue in full in 2034 . This useless work doesn't need to be done either.

Addition for the Mercury's perihelion. Consider again the cause for the excessive rotation of the perihelion of Mercury. As noted above, the luminal velocity of gravitation gives a perihelion rotation of $0.23 \mathrm{arcsec} /$ century [1], and not $43 \mathrm{arcsec} /$ century, i.e. in 187 times less. In addition, the luminal velocity of gravitation leads to changes in the eccentricity of the orbit, its semi-axis and the period of revolution of the same order as the perihelion rotation [3-5], [21-23]. With the Newtonian gravitational interaction, changes in all orbital parameters for all planets coincide with observations [12, 19], with the exception of the Mercury's perihelion. Therefore, the finite velocity of the propagation of gravitation in no way can be the reason for the excessive rotation of the Mercury's perihelion, because in this case other parameters of the orbit will not coincide with the observations.

So what is the real cause for the excessive rotation of the Mercury's perihelion?

In the above-mentioned works $[12,18,20]$, the oblate shape of the Sun was modeled by a ring of bodies around the Sun (Figure 2), while the mass of such compound model of the Sun was equal to its mass. At a certain mass of peripheral bodies, the total speed of rotation of the perihelion coincides with the
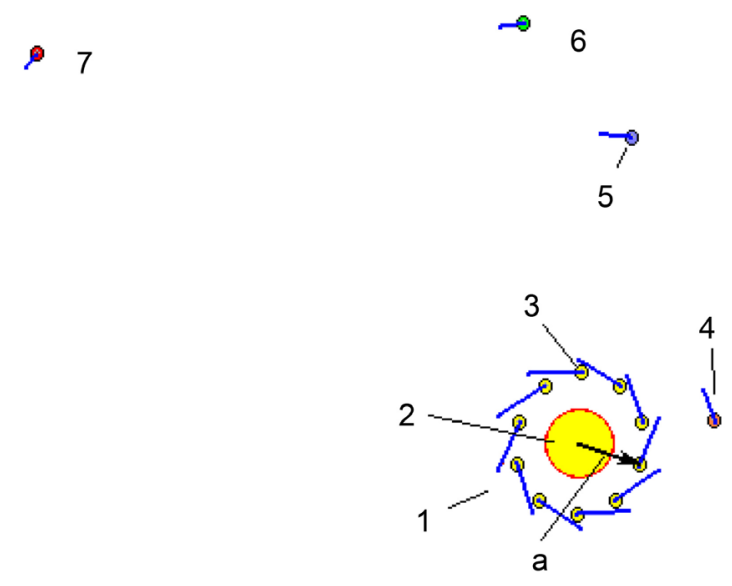

\begin{tabular}{|c|c|c|c|}
\hline \multirow{2}{*}{$\begin{array}{c}\text { No } \\
\text { of models }\end{array}$} & \multirow{2}{*}{$\mathrm{n}_{1}$} & $\mathrm{~m}_{1} \times 10^{-23}$ & $\mathrm{a} \times 10^{-10}$ \\
\cline { 3 - 4 } & & $\mathrm{kg}$ & $\mathrm{m}$ \\
\hline 2 & 5 & 604.8 & 2.528358 \\
\hline 3 & 5 & 1.966 & 2.528449 \\
\hline 4 & 5 & 1.565 & 2.528449 \\
\hline 5 & 10 & 0.782 & 2.528449 \\
\hline 15 & 5 & 3495.308 & 0.06742779 \\
\hline
\end{tabular}

Figure 2. The compound models for Sun's rotation and their parameters for the Sun mass $M_{S}=$ $1.98892 \times 10^{30} \mathrm{~kg}$ and radius $R_{S}=6.97113 \times 10^{8} \mathrm{~m}: 1$-compound model for the Sun; 2-central body; 3-peripheral body; 4-Mercury; 5-Venus; 6-Earth and Moon; 7-Mars. Other planets are outside the field of picture. The positions of the bodies are indicated for December 30, 1949. The line bars at the bodies indicate their velocity vectors. In table: $n_{1}$-number of peripheral bodies; a-radius of their orbit; $m_{1}-$ mass of a peripheral body. 
observed speed of 583 arcsec/century. At the same time, changes in all other parameters of the Mercury's orbit of and other planets coincide with observations.

The compound Sun model simulates the oblateness of the Sun. However, the bodies on the ring move with certain velocity in orbit around the Sun. Therefore, it may be assumed that this velocity affects the rotation of the perihelion. The compound model of the Sun was developed with half the velocity [12]. This model gave exactly the same rotation of the perihelion. That is, the result does not depend on the velocity of the bodies on the ring.

A model was also developed with half the masses of bodies on the ring [20]. It led to half the excess rotation of the perihelion. It should be noted that a total of 15 compound models of the Sun were created. In the latest models, the radius of the ring was equal to the equatorial radius of the Sun.

Therefore, the real cause for the excessive rotation of the Mercury's perihelion is the flattened shape of the Sun.

\section{DISCUSSION}

The above text has been reviewed by the reviewer. This is followed by the text of the review, in which the author has marked points by letters in parentheses. Below, in accordance with these points, the author presents his explanations and answers.

\subsection{Review}

\subsubsection{Summary}

The paper is concerned with a presentation and a review of two papers speaking about big bang model, dark matter, special relativity and gravitational waves. The author claims that the big bang model is wrong because the large red shift of quasars requires them to move faster than the speed of light in free space (A). He also said that no gravitational waves exist because they have been proposed to explain excess rotation of mercury perihelion. According to his view such perihelion can be explained using Newton's laws and assuming it resulting from the sun oblateness. He also claims that the numerical value of the excess rotation is $0.23 \mathrm{arcsec} / \mathrm{century}$ and not 43 . Thus it does not agree with observations (B). He thinks that special relativity itself suffers from some setbacks. He thinks that the explanation of charged particles interactions assuming change of particles masses (as proposed by special relativity) is wrong. It can be explained using velocity and spatial dependent force. He also thinks that no dark matter exists in reality or at least the nature of dark matter is misleading.

\subsubsection{General Comments}

The paper is written properly. Where the abstract is concerned with the methodology, results obtained. The introduction gives a brief survey of the studies indicating the set backs of the big bang model specifically that are concerned with the existence of gravitational waves and dark matter. Fluent English language was used to write the paper. However the claims of throwing a way the big bang model and relativity theory utilized weak and few evidences to support this claim.

\subsubsection{Constructive Criticism}

1) The suggestion that no gravitational waves exists because the mercury perihelion can be explained using Newton's laws and oblate sun is very weak. This is due to the fact that no derivation was made to confirm this claim. Such derivation is needed (C). Even if such derivation was made, this does not mean that no gravitational waves exist, because many other evidences from binary pulsars and black holes as that detected by LIGO on 2016 due to black holes collision confirm the existence of gravitational waves (D). Thus there is a need to explain these observations on new basis. Even the claim that calculated values of excess rotation is wrong needs more details. The author should write down the basic equations, and then show that the excess rotation value is wrong (E). Thus all these big bang predictions should be shown using basic laws to be incorrect. 
2) There are many suggestions proposed by many authors to confirm the existence of dark matter or finding a new path ways to remove the conflict of observed and predicted actual and critical densities of the universe, without abandoning the big bang model. One can refer to Arbab paper (Astrophy. Space. Sci(2014) 355, or M. Dirar (Modern Physics Letters A, V. 13, N.37(1998) to see some of these models. Thus the critical and present mass density problem needs also more strong evidences to confirm the failure of the big bang model (F).

3) The claim that the interaction of charged particles can be explained assuming constant mass and using a new force expression is very hard to be justified. The postulates on which this new force expression is found needs to be mentioned and written properly $(G)$. The experimental observations that confirm the fact that the particles masses change like nuclear fission and fusion beside pair production and particles annihilation make it difficult to believe that the particle masses are constant physical quantities (H). This needs to be explained using the new force expression or any other model (I).

4) The very brief information extracted from the cited references forces readers to refer to these references, which is time consuming. From the physical stand point, it is preferable for the authors to spend more time to give more details so as to gain time for all readers of this manuscript.

\subsection{Author's Response}

A) Here it is need to clarify, why the Big Bang theory is wrong

Any incorrect statement has multiple contradictions with reality. Some of them are main or initial, and the second are their consequences. Let's note the main contradictions.

First, there is no reason for the birth of the Universe from a point, i.e. there is no reason that could create such an explosion of a point.

Secondly, in a bang, the expansion velocities should decrease with increasing distance. Therefore, the interpretation of the red shift by the Doppler Effect is incorrect, because with this interpretation of the observations velocities increase with distance. Therefore, there is no expanding Universe and there is no Big Bang.

B) Gravitational waves follow from the theory of gravitational action, which propagates at the speed of light. An analogue of such action is an electromagnetic one. Therefore, it is assumed that, like electromagnetic waves, there must be gravitational waves. In the Theory of Relativity, by analogy with electromagnetic interaction, equations are created for the gravitational interaction propagating with the speed of light. For such an interaction, the excess of rotation of the perihelion of Mercury is 0.23 arcsec/century, not $43 \mathrm{arcsec} / \mathrm{century}$. The value $43 \mathrm{arcsec} /$ century obtained by Paul Geber [17] is incorrect. And according to observations, the excess of rotation of the perihelion of Mercury is 53 arcsec/century, and not 43 arcsec/century.

C) Excessive rotation of the perihelion of Mercury 53 arcsec/century due to the Sun's oblateness was established as a result of the numerical solution of the problem of the Newtonian interaction of the compound model of the Sun (Figure 2) and Mercury for \pm 3000 years from the modern epoch [20]. The complete rotation of the perihelion of Mercury 583 arcsec/century was too established as a result of the numerical solution of the problem of the interaction of the planets of the Solar system and the compound model of the Sun (Figure 2) for \pm 3000 years from the modern epoch [12, 18]. At the same time, secular changes in all parameters of the orbits of Mercury and other planets were determined. They were being compared with observations. The calculation results coincide with observations [12].

These works were carried out for two decades. The results have been published in dozens of papers and in several monographs. This material is huge, and it is impossible to give it in this paper.

D) The signals detected in 2016 are not evidence of gravitational waves [2]. B.P. Abbott et al. have described results of their observation of gravitational waves emitted as a result of a collapse of a double Black hole [14]. This is the so-called LIGO experiment.

On September 14, 2015 at 9:50:45 the employed device had recorded a pulse with several oscillations, which lasted about 0.15 seconds ... the fusion of the black holes had imparted the test body with acceleration of $10^{-21} \mathrm{~cm} / \mathrm{sec}^{2}[14]$. 
The first point. The reader can imagine that the equipment used to perform the LIGO experiment should register test-body displacements as small as $1.1 \times 10^{-23} \mathrm{~cm}$. The diameter of the hydrogen atom is $0.529 \times 10^{-8} \mathrm{~cm}$. Therefore, it can be said that, for performing the LIGO experiment, an apparatus was created that allows measuring lengths constituting $2 \times 10^{-15}$ fraction of the atom size.

The second point. The collision of the black holes and their merging together had occurred at a distance of 410 mega parsecs. This distance was determined with an accuracy of -180 to +160 mega parsec [14]. If expressed in light years (l.y.), this distance is 1.34 billion l.y., lying somewhere in the interval from -0.59 to +0.52 billion l.y.

Since gravitational waves propagate at the speed of light, the fusion of black holes could happen within a time interval from 590 million years ago till 520 million years in the future (!).

The third point. The collision of two objects of size $D$ moving at velocity $v$ occurs in a time $t=D / v$. The pulse from their collision will have approximately the same duration $t$. For example, a collision of two bullets normally occurs in a time of $t=60$ microseconds, and the collision of two ocean-going liners, in a time of $t=0.5$ minutes. Such will also be the duration of the collision-generated pulses. Consider now the collision time of two galaxies, with black holes residing at their centers. The standard diameter of galaxies is $D=10^{5}$ l.y., and their relative velocity is $V=1000 \mathrm{~km} / \mathrm{sec}$. We will take into account the fact that one light-year is equal to $9.461 \times 10^{12} \mathrm{~km}$. Then, the collision time can be evaluated as $t=30$ million years.

So, the duration of the pulse produced by two merging galaxies moving at a relative velocity of 1000 $\mathrm{km} / \mathrm{sec}$ is $t=30$ million years, and not $t=0.15 \mathrm{sec}$, which time was registered in the LIGO experiment.

Suppose that LIGO scientists are right, and they have indeed detected a collision of two black holes. Let us evaluate the velocity of the colliding black holes; for this velocity, we obtain a value $v=D / t=21000$ billion $\mathcal{C}$, , where $c$ is the speed of light.

That is, in the LIGO experiment, the galaxies moved at velocities exceeding the speed of light by twenty-one thousand billion times.

The Theory of Relativity is based on the assumption that nobody can move faster than light. The publication of the LIGO experiment was timed to coincide with the 100th anniversary of the General Theory of Relativity to allegedly confirm this theory.

Evidently, the LIGO experiment has in fact disproved the Theory of Relativity. Therefore, based on this "discovery of the century" in the mainstream science, once again I say: the Theory of Relativity and the entire modern physical picture of the micro- and macro-world must be thrown away and forgotten!

E) The actual excess of rotation of Mercury's perihelion of $0.23 \mathrm{arcsec} / \mathrm{century}$ was determined as follows [1]. By analogy with the electromagnetic action, the force of the gravitational interaction propagating at the speed of light has the following form:

$$
\boldsymbol{F}_{i k}=-\frac{G m_{i} m_{k}\left(1-\beta_{i k}^{2}\right) \boldsymbol{r}_{i k}}{\left\{r_{i k}^{2}-\left[\boldsymbol{\beta}_{i k} \times \boldsymbol{r}_{i k}\right]^{2}\right\}^{3 / 2}}
$$

where $G$ is the gravitational constant;

$m_{i}$ and $m_{k}$ are the masses of the $i$-th and $k$-th bodies; other designations are the same as for force (1).

The problem of interaction of two bodies with force (2) was solved. The trajectory equation is obtained in the following dimensionless form:

$$
\phi=\int \frac{\mathrm{d} \bar{R}}{\bar{R}^{2} \bar{v}_{r}},
$$

where $\bar{v}_{r}=v_{r} / v_{p}$ is the dimensionless radial velocity of Mercury relative to the Sun, which is determined by the equation:

$$
\bar{v}_{r}=\frac{1}{\beta_{p}} \sqrt{1-\frac{\beta_{p}^{2}}{\bar{R}^{2}}-\left(1-\beta_{p}^{2}\right) \exp \left[2 \alpha_{1} \beta_{p}^{2}\left[\frac{1}{\sqrt{\bar{R}^{2}-\beta_{p}^{2}}}-\frac{1}{\sqrt{1-\beta_{p}^{2}}}\right]\right]}
$$


$\bar{R}=r / R_{p}$ is dimensionless distance from the Sun to Mercury;

$\beta_{p}=v_{p} / c_{1}$ is the velocity of Mercury at perihelion, reduced to the speed of light;

$R_{p}$ and $v_{p}$ are the radius of the orbit perihelion of the Mercury and its velocity at perihelion;

$\alpha_{1}=-G\left(m_{S}+m_{M e} /\left(R_{p} v_{p}^{2}\right)\right.$ is trajectory parameter;

$m_{S}$ and $m_{M e}$ are the masses of the Sun and Mercury.

The trajectory Equation (3) is given in the polar coordinate system $(r, \varphi)$, in the center of which the Sun is located, and the polar angle $\varphi$ is measured from the perihelion. For the interaction of the Sun and Mercury $\alpha_{1}=-0.829$ and $\beta_{p}=1.96 \times 10^{-4}$.

The trajectory Equation (3) - (4) cannot be solved by an exact analytical method. Its solution by approximate analytical methods at very small values $\beta_{p}$ is difficult due to the presence of singularities at the end points, i.e. at perihelion and aphelion.

The trajectory Equation (3) - (4) was solved numerically with variation $\alpha_{1}$ and $\beta_{p}$ in the entire range of their variation [21-23]. Equation (3) was integrated from the perihelion radius to the aphelion radius. Based on these numerical solutions, an asymptotic solution was obtained. For $\alpha_{1}=-0.829$ and small $\beta_{p}$, the displacement of the perihelion of Mercury for its full revolution in orbit is

$$
\Delta \varphi=1.6 \pi \beta_{p}^{2.5} .
$$

For 100 Earth's years, Mercury makes 415 revolutions, which, according to (5), gives a displacement of the perihelion of Mercury of 0.23 arcsec/century.

F) As shown in point A, the Big Bang hypothesis is wrong. Therefore, the entire interpretation of phenomena in the macrocosm using this hypothesis is incorrect, and it is necessary to get rid of it.

G) Expression (1) for the force of action on a particle with a charge $q_{1}$ of another particle with a charge $q_{2}$ is obtained on the basis of the experimental laws of electromagnetism [21-23]. The second particle moves at a velocity $\boldsymbol{v}=\boldsymbol{v}_{i k}$ relative to the first one. Therefore, its motion is identical to the current $I$, which, in accordance with the Biot-Savart-Laplace law, creates a magnetic field with a strength $H$ at the point where the first particle is located. Since the second particle is moving, the field $H$ is variable. In accordance with Faraday's law of induction, an alternating magnetic field $H$ at the point where the first particle is located creates an electric field with a strength $E$. By definition, the strength $E$ is the force acting on the first particle with a charge equal to a unit. These two laws are differential equations. Eliminating the strength $H$ from them, we obtain the following differential equation for the force of interaction of a moving charge $q_{2}$ on a motionless charge $q_{1}$ :

$$
\frac{\partial^{2} \boldsymbol{F}}{\partial x^{2}}+\frac{\partial^{2} \boldsymbol{F}}{\partial y^{2}}+\frac{\partial^{2} \boldsymbol{F}}{\partial z^{2}}-\frac{1}{c_{1}^{2}} \frac{\partial^{2} \boldsymbol{F}}{\partial t^{2}}=\frac{4 \pi q_{1}}{\varepsilon}\left[\frac{1}{c_{1}^{2}} \frac{\partial(\rho \boldsymbol{v})}{\partial t}+\operatorname{grad} \rho\right],
$$

where $\rho$ is the charge density $q_{2}$ distributed over space.

As a result of solving Equation (6) for a point charge $q_{2}$, an equation for the force (1) is obtained [21], [23]. The solution to Equation (6) is a significant achievement in mathematics. It was received by the author in 1968.

H) There are many incomprehensible phenomena in the microcosm. However, the introduction of hypothetical mechanisms of phenomena does not contribute to a clearer understanding of the surrounding world. The introduction of hypotheses leads to new contradictions and ambiguities. As a result, we come to a completely imaginary and fantastic world.

I agree with the reviewer that modern interpretation of experimental observations makes it difficult to believe that the particle masses are constant physical quantities. However, this interpretation was created on the presumption that the mass of one particle changes depending on its relative velocity to another particle. In fact, as shown above, there is no reason for a change in mass. The dependence of mass on velocity is an incorrect position. Like any wrong statement, it contradicts reality in many ways. I have cited one of these contradictions above. A particle in relation to other particles moves at different velocities. Therefore, a particle at the same moment must have as many masses as there are particles in the world.

These contradictions confirm that the hypothesis about the change in mass with velocity is erroneous. 
Therefore, this statement must be thrown out from science. It is also need to discard the entire structure of the microworld built on this presumption.

For 100 years of building the world around with the help of hypotheses, several generations of physicists have changed. Introduced imaginary objects by their first generations, by subsequent generations were perceived as real objects. Now everything that supposedly exists in the surrounding world is taken for granted by the majority. Therefore, the entire contemporary structure of the micro- and macrocosm needs to be revised today. As an example, I will give the history of the creation of neutrinos [23].

Reckardo Cerezani and David de Hilster in their works [24-26] clearly outlined the situation with neutrinos. Radioactive radium converts to polonium and emits electrons. In 1927, C.D. Ellis and W.A. Wooster [27] measured the electron energy with a calorimeter and obtained its value $0.36 \mathrm{MeV}$. In 1931, Wolfgang Pauli calculated the kinetic energy of an electron from the standpoint of the Theory of Relativity and received $1.16 \mathrm{MeV}$. This value is due to the dependence of the electron mass on the velocity, accepted in the Theory of Relativity. In reality, the mass is unchanged, and the force depends on the velocity. However, relativists believe that such an erroneous energy of $1.16 \mathrm{MeV}$ is real, so they invent a neutrino particle, which should carry a false energy of $1.16 \mathrm{MeV}-0.36 \mathrm{MeV}=0.86 \mathrm{MeV}$.

For more than half a century, science has been conducting grandiose experiments to search for neutrinos. It is not known how it can be found, since a neutrino without interaction can pass through both the Earth and the Sun. In different radioactive transformations, different neutrinos with different energies must be added. Nevertheless, neutrinos have now been introduced into all nuclear reactions. All their energy balances have been experimentally measured. Theorists increased the experimental values by the fictitious energy of neutrinos, and immediately they are reduced by this value, due to the fact that all neutrinos freely fly away.

I) Using force (1), interactions of a moving charged particle with charged bodies of various shapes, with conductors with current and with magnets were considered [1]. The theory of particle accelerators has been developed. It is shown that calculations using force (1) are more accurate than those based on the Theory of Relativity. For example, in the experiment of Bucherer [28] at the velocity of electrons approaching the speed of light, the Theory of Relativity gives an incorrect result. And the calculations using the force (1) completely coincide with the results of this experiment [21, 23].

\section{CONCLUSION}

Now such words can be heard from the followers of the Big Bang. The Big Bang might not have happened. Perhaps the Universe is constantly "shrinking" and "expanding". Or maybe the Universe is infinite, but space and time have always existed. But if there was no Big Bang, what was instead?

This is the result of fundamental research that has been going on for 100 years. The fictional world raises doubts even among its creators.

Now fundamental science is faced with the task not of creating a new surrounding world, but of analyzing the constructed ideas about the micro- and macrocosm. It is necessary in these constructions to reveal unreasonable hypotheses and remove the chains of imaginary constructions associated with them from science. By consistently doing this work, we will receive real knowledge about the world around us.

I encourage novice researchers to engage in this work, and not get carried away with the creation of grandiose structures from hypotheses, which will disappoint them with their meaninglessness by the end of their lives.

\section{ACKNOWLEDGEMENTS}

I express my gratitude to the reviewer for the thorough analysis of the manuscript, his advices and questions.

\section{CONFLICTS OF INTEREST}

The author declares no conflicts of interest regarding the publication of this paper. 


\section{REFERENCES}

1. Smulsky, J.J. (2004) The Theory of Interaction. Cultural Information Bank, Ekaterinburg. http://www.ikz.ru/ smulski/TVEnA5_2.pdf

2. Smulsky, J.J. (2019) New Understanding in Academic Science. Natural Science, 11, 74-94. https://www.scirp.org/journal/PaperInformation.aspx?PaperID=91189 https://doi.org/10.4236/ns.2019.113009

3. Smulsky, J.J. (2002) The New Fundamental Trajectories: Part 1-Hyperbolic/Elliptic Trajectories. Galilean Electrodynamics, 13, 23-28.

4. Smulsky, J.J. (2002) The New Fundamental Trajectories: Part 2-Parabolic/Elliptic Trajectories. Galilean Electrodynamics, 13, 47-51. http://www.ikz.ru/ smulski/smul1/English1/FounPhisics/NFT.pdf

5. Smulsky, J.J. (1994) The New Approach and Superlight Particle Production. Physics Essays, 7, 153-166. http://www.ikz.ru/ smulski/smul1/English1/FounPhisics/NApSup.pdf https://doi.org/10.4006/1.3029128

6. Smulsky, J.J. (2003a) Axisymmetrical Problem of Gravitational Interaction of N-Bodies. Mathematical Modelling, 15, 27-36. (In Russian) http://www.ikz.ru/ smulski/smul1/Russian1/IntSunSyst/Osvnb4.doc

7. Smulsky, J.J. (2015b) Exact Solution to the Problem of N-Bodies Forming a Multilayer Rotating Structure. SpringerPlus, 4, Article No. 361. http://www.springerplus.com/content/4/1/361 https://doi.org/10.1186/s40064-015-1141-1

8. Smulsky, J.J. (2019) Periodic Orbits of N Bodies on a Sphere. Cosmic Research, 57, 459-470. http://link.springer.com/article/10.1134/S001095251906008X

https://rdcu.be/b03dI https://doi.org/10.1134/S001095251906008X

9. Smulsky, J.J. (2017) Advances in Mechanics and Outlook for Future Mankind Progress. International Journal of Modern Education and Computer Science, 9, 15-25. https://doi.org/10.5815/ijmecs.2017.01.02 http://www.mecs-press.org/ijmecs/ijmecs-v9-n1/IJMECS-V9-N1-2.pdf

10. Smulsky, J.J. (2012) Galactica Software for Solving Gravitational Interaction Problems. Applied Physics Research, 4, 110C123. http://www.ikz.ru/ smulski/Papers/Galct12E2J.pdf https://doi.org/10.5539/apr.v4n2p110

11. Smulsky, J.J. (2012) The System of Free Access Galactica to Compute Interactions of N-Bodies. International Journal of Modern Education and Computer Science, 4, 1-20. https://doi.org/10.5815/ijmecs.2012.11.01

12. Smulsky, J.J. (2018) Future Space Problems and Their Solutions. Nova Science Publishers, New York, 269 p. http://www.ikz.ru/ smulski/Papers/InfFSPS.pdf

13. Smulsky, J.J. (1996) The "Black Hole": Superstition of the 20th Century. Apeiron, 3, 22-23. http://www.ikz.ru/ smulski/smul1/English1/FounPhisics/BHAP2.doc

14. Abbott, B.P., et al. (2016) Observation of Gravitational Waves from a Binary Black Hole Merger. Physical Review Letters, 116, Article ID: 061102. https://doi.org/10.1103/PhysRevLett.116.061102

15. Smulsky, J.J. (2016) On Performance Indicator of Scientist's Scientific Work. The Way of Science. International Scientific Journal, 10, 8-16. (In Russian) http://www.ikz.ru/ smulski/Papers/PokRezNauchD4.pdf

16. Smulsky, J.J. (2019) The Upcoming Tasks of Fundamental Science. Sputnik+ Publishing House, M., 134 p. (In Russian) http://www.ikz.ru/ smulski/Papers/InfPrZaFN.pdf

17. Gerber, P. (1898) Die raumliche und zeitliche Aubreitung der Gravitation. Zeitschrift für Mathematik und Phy- 
sik, 43, 93-104. http://bourabai.narod.ru/papers/gerber/gerber.htm

18. Smulsky, J.J. (2011) New Components of the Mercury's Perihelion Precession. Natural Science, 3, 268-274. http://www.scirp.org/journal/ns https://doi.org/10.4236/ns.2011.34034

19. Smulsky, J.J. and Smulsky, Ya.J. (2012) Dynamic Problems of the Planets and Asteroids, and Their Discussion. International Journal of Astronomy and Astrophysics, 2, 129-155. https://doi.org/10.4236/ijaa.2012.23018

20. Smulsky, J.J. (2008) Numerical Modeling of Evolution of the Satellite of a Rotating Body. In: Col. The Theoretical and Applied Tasks of the Nonlinear Analysis, Russian Academy of Sciences: A.A. Dorodnicyn Computing Center, Moscow, 100-117. (In Russian) http://www.ikz.ru/ smulski/Papers/ModSun07c.pdf

21. Smulsky, J.J. (1994) The Electromagnetic and Gravitational Actions (The Non-Relativistic Tractates). "Science" Publisher, Novosibirsk, 225 p. (In Russian) http://www.ikz.ru/ smulski/smul1/English1/FounPhisics/ELGRVZIN.doc

22. Smulsky, J.J. (1995) The Trajectories at Interaction of Two Bodies, Which Depend on Relative Distance and Velocityю Mathematical Modeling. Mathematical Modeling, 7, 111-126. (In Russian) http://www.ikz.ru/ smulski/smul1/English1/FounPhisics/ELGRVZIN.doc

23. Smulsky, J.J. (2014) Electrodynamics of Moving Bodies. Determination of Forces and Calculation of Movements. Palmarium Academic Publishing, Saarbrucken, 324 p. (In Russian) http://www.ikz.ru/ smulski/Papers/InfElMvBEn.pdf

24. Carezani, R.L. (1997) Nuclear-Nuclear Collisions.

25. Carezani, R.L. (1997) Neutrinos at Fermi Lab.

26. de Hilster, D. (2011) The Neutrino: Doomed from Inception. Proceedings of the NPA, 8, 148-151.

27. Ellis, C.D. and Wooster, W.A. (1927) The Average Energy of Disintegration of Radium E. Proceedings of the Royal Society London A, 117, 109-123. https://doi.org/10.1098/rspa.1927.0168

28. Bucherer, A.H. (1909) Die experimentelle Bestatigung des Relativitats Prinzips. Annalen der Physik, 28, 513 S. https://doi.org/10.1002/andp.19093330305 